\title{
ALGORD : un logiciel pour apprendre à apprendre une langue vivante
}

Jean-Pierre Soula, Anne Hernandez et Daniel Marre

\section{CpenEdition}

\section{Journals}

Édition électronique

URL : http://journals.openedition.org/asp/4405

DOI : 10.4000/asp.4405

ISSN : 2108-6354

Éditeur

Groupe d'étude et de recherche en anglais de spécialité

Édition imprimée

Date de publication : 1 mars 1993

Pagination : $547-549$

ISSN : 1246-8185

Référence électronique

Jean-Pierre Soula, Anne Hernandez et Daniel Marre, « ALGORD : un logiciel pour apprendre à apprendre une langue vivante », ASp [En ligne], 1 | 1993, mis en ligne le 04 juin 2014, consulté le 19 avril 2019. URL : http://journals.openedition.org/asp/4405; DOI : 10.4000/asp.4405

Ce document a été généré automatiquement le 19 avril 2019

Tous droits réservés 


\title{
ALGORD : un logiciel pour apprendre à apprendre une langue vivante
}

\author{
Jean-Pierre Soula, Anne Hernandez et Daniel Marre
}

1 ALGORD est un logiciel conçu comme outil pour apprendre une langue vivante, d'où son nom « Apprentissage de Langues Géré par ORDinateur ». Il prend la forme d'un classeur informatique qui accompagne l'apprenant tout au long de son étude, favorisant l'acquisition et la révision de ses connaissances linguistiques.

2 ALGORD n'est pas un système "clef en main "; il ne propose pas un ensemble prédéfini et/ou fini de données. Au contraire, c'est l'apprenant lui-même qui va les fournir car le système est ouvert et individuel. L'apprenant est ainsi impliqué activement et ce sont les démarches qui lui sont proposées dans l'utilisation du logiciel qui font qu'ALGORD devient aussi un outil pour apprendre à apprendre une langue.

\section{Positionnement fonctionnel}

3 Avant de décrire ces différentes démarches, il est intéressant de situer visuellement ALGORD par rapport à d'autres logiciels d'E.A.O. Pour le faire, nous avons construit une grille de positionnement fonctionnel ${ }^{1}$ qui prend en compte les objectifs visés par un didacticiel et les ressources humaines ou matérielles qu'il met en œuvre (voir figure 1).

Chaque logiciel est caractérisé par un symbole qui est placé dans la case correspondante aux caractéristiques du logiciel. On peut ainsi se rendre compte qu'algord se situe dans une zone qui privilégie "l'ouverture » et se démarque ainsi des logiciels qui apportent un contenu lexical ou grammatical clos. Il exploite une partie des possibilités que donnent les logiciels de gestion de fichiers. 
Figure 1. Grille de positionnement fonctionnel

\begin{tabular}{|c|c|c|c|c|c|c|c|c|c|c|c|}
\hline & $\begin{array}{l}\text { TRRVACL } \\
\text { CNDWLDUEL }\end{array}$ & $\begin{array}{l}\text { TRQVOALL } \\
\text { GROUPE }\end{array}$ & $\begin{array}{l}\text { ACCES } \\
\text { FERME }\end{array}$ & $\begin{array}{l}\text { OUVERT } \\
\text { FROF }\end{array}$ & $\begin{array}{l}\text { OUVERT } \\
\text { MPPPREN } \\
\text { MNT }\end{array}$ & $\begin{array}{l}\mathrm{CD} \\
\mathrm{ROM}\end{array}$ & IMAGE & SON & $\begin{array}{l}\text { FCT. } \\
\text { EQSEE } \\
\text { DONNEES }\end{array}$ & $\begin{array}{l}\text { FCT. } \\
\text { TRALT. } \\
\text { TEQT }\end{array}$ & \begin{tabular}{|l} 
FCT \\
TMELLE \\
UR
\end{tabular} \\
\hline GRAMMAIRE & $\begin{array}{l}\mathrm{CN} \\
\mathrm{E} \\
\mathrm{EE}\end{array}$ & & $\begin{array}{l}\mathrm{CN} \\
\mathrm{EE}\end{array}$ & & & & EE & E & & & \\
\hline VOCABULAIRE & $\begin{array}{l}\text { E } \\
\text { EE }\end{array}$ & & EE & & & & EE & E & & & \\
\hline TRADUCTION & EE & & $E B$ & & & & $E E$ & E & & & \\
\hline ECR. СOMP. & & & & E & & & & E & & & \\
\hline ECR. PROD. & & D & & & & & D & & & D & \\
\hline ORAL COMP. & EB & & EB & & & & EE & & & & \\
\hline ORAL PROD. & & D & & & & & D & & & & \\
\hline
\end{tabular}

V. Patureau nous interpelle dans la conclusion de son article (in Duda \& Riley $1990: 126$ ) sur « Styles d'apprentissage et ordinateurs », en nous disant que

l'ordinateur au service de la formation nous lance un enjeu: le veut-on outil à reproduire ce qui s'est toujours fait dans l'enseignement ou outil pour dynamiser les actes d'apprendre et d'enseigner pour travailler avec les modes d'apprendre diversifiés d'aujourd'hui et former les hommes autonomes dont nous aurons besoin demain.

6 Nous nous rangeons résolument dans la deuxième proposition et nous suggérons qu'ALGORD contribue à dynamiser les actes d'apprentissage et à former des individus autonomes.

\section{Autonomie, responsabilité}

7 L'autonomie, comme le souligne H. Holec, n'est pas innée et ne s'acquiert que par l'entraînement :

Teaching must also help the learner acquire autonomy for him/herself, i.e. to learn to learn. (Holec 1981 : 23)

Cela consiste à réduire la traditionnelle dépendance totale de l'apprenant envers l'enseignant en l'encourageant à prendre une plus grande responsabilité dans son apprentissage. Pour y parvenir, il a besoin qu'on l'aide à découvrir et affiner ses propres stratégies. Explorer des stratégies différentes l'amenera à mieux choisir celles qui lui conviendront personnellement.

9 Algord conduit l'apprenant vers cette prise de responsabilité. Il a été conçu comme outil d'apprentissage: «for LEARNING not for TEACHING». Il a la forme d'un classeur personnel, doublé d'un exerciseur. Sa structure générale se présente ainsi (voir figre2). 
Figure 2

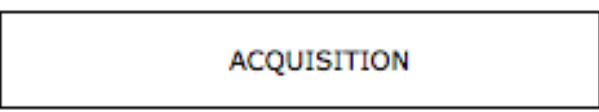

CLASSEUR

\begin{tabular}{|c|c|c|c|c|}
\hline VOCAB & $\begin{array}{l}\text { VOCAB. } \\
\text { SPECIA. }\end{array}$ & GRAMM. & $\begin{array}{c}\text { MOTS } \\
\text { OUTILS }\end{array}$ & $\begin{array}{c}\text { STRUCT. } \\
\text { UTILIT. }\end{array}$ \\
\hline
\end{tabular}

EXERCISEUR

REVISION

10 Au départ, le classeur est vide ; l'apprenant décide lui-même ce qu'il y met et comment il l'utilise. C'est, en fait, un outil d'accompagnement de son étude de l'anglais ${ }^{2}$ (qu'elle soit faite en cours traditionnels ou en auto-apprentissage) et est destiné à aider l'apprenant à structurer ses connaissances.

11 L'apprenant fait donc ses propres entrées lexicales et grammaticales. Il doit également procéder aux vérifications de ses données (par exemple la phonétique et la traduction des mots ou expressions, les règles de grammaire). Ensuite, il trouve ses propres phrases d'illustration et réalise lui-même leur traduction. L'obligation d'enregistrer les données et de les vérifier lui-même l'implique dans son apprentissage et joue également un rôle important dans la mémorisation.

12 En utilisant Algord, l'apprenant est invité à procéder par étapes, ce qui peut l'amener à mettre en place des mécanismes dans sa façon d'apprendre. C'est à lui de réfléchir sur les éléments lexicaux et grammaticaux qu'il souhaite retenir, les caractéristiques qui vont l'aider à les retrouver quand il en aura besoin. Comme l'explique J. Rubin (in Wenden \& Rubin 1987 : 22) dans son travail sur «Learner strategies », pour certains apprenants, ce «conscious attention to the learning process is the first step to making language automatic ».

\section{Organisation}

Algord pousse l'apprenant à adopter l'un des comportements du «good language learner " en l'encourageant à s'organiser. Cette organisation intervient à plusieurs stades. 


\section{Figure 3} VOCABULAIRE GENERAL

La première option concerne la phonétique. L'apprenant rentre un mot (ici, «attic »). S'il le souhaite, il peut enregistrer le symbole phonétique de la syllabe accentuée. Dans ce cas, une liste apparait automatiquement et il fait son choix. Dans le cas contraire, il passe directement à la traduction.

Ensuite vient le classement par thèmes et sous-thèmes. Certains sont suggérés, mais l'apprenant peut créer les siens propres ou même supprimer ceux que nous avons proposés. Une autre possibilité est de remettre ce classement à plus tard en utilisant la boîte « divers ».

Enfin, il peut ou non formuler une phrase d'illustration du mot saisi. L'apprenant devrait donc prendre l'habitude de s'organiser à l'intérieur d'une structure qui laisse de la place à ses choix personnels.

\section{Auto-évaluation}

G. Ellis et B. Sinclair (1989) et d'autres défenseurs qui plaident en faveur du «learner training " soulignent l'importance d'une auto-évaluation régulière. L'apprenant peut ainsi évaluer ses progrès, réfléchir sur l'efficacité de ses stratégies et être en mesure de décider de son prochain objectif.

Grâce à l'exerciseur d'ALGORD, il peut tester régulièrement ses connaissances; ses scores, qui sont enregistrés, lui permettent de mesurer ses progrès. Les exercices sont créés à partir des données portées sur les différentes fiches : il y a des exercices de phonétique, de traduction, d'appariement, de phrases à trous, de phrases à trous guidées.

Cependant, avec un tel système dans lequel l'apprenant lui-même enregistre les données, se pose la question de leur correction. Une approche de solution sera un dictionnaire caché qui permettra de vérifier l'orthographe, le positionnement de l'accent tonique ainsi que le symbole phonétique qui s'y rattache. Dans le cas d'une utilisation institutionnelle, 
l'enseignant pourrait, de temps en temps, y jeter un regard et apporter des corrections nécessaires.

Des logiciels vérificateurs de structures tels Hugo plus pourraient peut être apporter un complément de solution, mais cela n'est qu'une réponse partielle à cet écueil.

Enseigner/apprendre conduisent à opérer des choix puisque les neuro-psycho-linguistes nous ont appris qu'il y a un «trade-off» entre "fluency » et « accuracy». Ce que l'on gagne d'un côté, on le perd de l'autre et vice-versa, tant que la compétence langagière n'est pas bien installée.

Avec ALGORD, nous nous rangeons plutôt du côté de la "fluency» car nous sommes conscients que notre logiciel ne peut générer informatiquement l'«accuracy». Nous pensons que celle-ci devrait l'être en amont puisque l'apprenant, conscient de l'enjeu devrait être rigoureux dans son travail de préparation. Nous partageons en cela les points de vue de R. Gairns et S. Redman qui recommandent

The most helpful guidance teachers can give here is to show learners how to be systematic whatever system they adopt. Being thorough about the information they record is one way. (1990:9)

Peut-être, sommes-nous trop optimistes, mais l'expérimentation que nous menons à l'I.N.S.A. nous permettra sans doute de mesurer l'étendue et les limites de la prise de responsabilité.

\section{La stratégie d'apprentissage académique}

Nous avons parlé au début de cet article de l'importance de l'acquisition de stratégies d'apprentissage. H. H. Stern (1983), dans ses études sur «the good language learner », a identifié quatre ensembles de stratégies :

- an active planning strategy

- an explicit academic learning strategy

- a social learning strategy

- an effective strategy

La situation d'auto-apprentissage est favorable au développement des deux premiers types de stratégies. Nous nous intéresserons plus particulièrement à la seconde, la stratégie d'apprentissage académique, que L. Dickinson résume ainsi :

Good language learners are able to view a language as a formal system with rules and regular relationships between language forms and meanings. They analyse the language and develop the necessary techniques of practice and memorisation. They monitor their own performance and revise it in order to progress towards an improved second language command. (1987:23)

Elle contient, en effet, trois éléments que nous considérons comme importants dans l'utilisation d'ALGORD, même si nous les traduisons en termes légèrement différents dans les paragraphes suivants.

\section{Vision personnelle du monde}

Un logiciel de langues permet d'habitude de travailler tel aspect grammatical ou tel aspect lexical et, en général, ne déborde pas de son objectif premier. ALGORD permet à l'apprenant de travailler ces cibles, mais il peut aussi s'adapter à la vision du monde de chacun. En effet, il invite l'utilisateur à procéder à des classements, à une hiérarchisation 
des données qui lui est personnelle. Les opérations que ce dernier est amené à faire s'inscrivent dans la ligne de l'« experiential learning » décrit par V. Kohonen :

Experiential learning aims to support the development of the learner's personality by increasing [..] his awareness of his own values and ways of categorizing the world. (in Duda \& Riley1990 : 35-36).

31 La catégorisation du monde décrite par V. Kohonen est sans doute de portée beaucoup plus générale que le découpage du monde auquel l'apprenant procède quand il classe ses propres données lexicales, mais l'opération découle de la même démarche: faire agir celui qui apprend sur ce qu'il apprend pour que, grâce à cette action, grâce aux choix opérés en fonction de sa propre expérience et de son vécu, l'« input » se personnalise, devenant un «throughput », avant d'être utilisé en « output » en passe d'être « maîtrisé ». «Meaningful output » nous dit encore V. Kohonen,

is based on 'digested' input which is somehow modified by the learner and thereby becomes his own.

\section{Perception de la langue en tant que système}

Le travail d'organisation des données en catégories joue à la fois sur la segmentation et sur la recomposition. Segmentation de la langue puisque l'utilisateur d'ALGORD est conduit à décomposer la langue selon plusieurs aspects, à entrer des mots, des règles de grammaire. Il est également poussé à recréer le contexte d'utilisation et ainsi s'amorce le processus de recomposition.

33 Cette reconstruction se poursuit au niveau de la boîte des «mots outils » ou mots de liaison, qui permet d'engranger les articulations du discours, ainsi que celui de la boîte de « structures utilitaires » que G. Ellis et R. Gairns appellent " patterns ».

Les phases d'analyse et de synthèse devraient montrer à l'utilisateur qu'une langue n'est plus un chaos de données que l'on ne sait pas très bien comment aborder, mais une série d'ensembles, de sous-ensembles interconnectés qui s'érige en un système.

Sans vouloir jouer sur les mots, nous pourrions faire l'hypothèse que l'approche « systématique » à laquelle invite ALGORD donne à l'utilisateur une vue « systémique » de la langue. Avoir touché la langue par clavier et écran interposés par le biais de petites unités que l'on aura situées sur la carte de la langue, manipulées et par là même maîtrisées jusqu'à un certain point devrait rassurer et mettre en confiance. La "réification» que peuvent donner le clavier et l'écran (mais que ne donnent pas nécessairement le crayon et le papier) pourrait sans doute faciliter le passage de la « compétence » à la « performance ».

\section{Jeu de la mémorisation}

Les psychologues ont montré que si les informations qui doivent être mémorisées sont mises en ordre, la personne peut utiliser cette organisation à son avantage : autrement dit, un matériel organisé est plus facile à stocker et à retrouver dans la mémoire à long terme. Voici pourquoi S. McDonough suggère que

the teacher's task is not so much to arrange for the repetition or recycling of L2 vocabulary, phrases or structural patterns but to help the student discover strategies for organizing his or her own knowledge. (1981: 73-74) 
Si ALGORD est un outil pour l'apprenant, il l'est également pour nous enseignants. Lors
des expériences menées auprès des élèves-ingénieurs de l'I.N.S.A., grâce aux capteurs
intégrés au logiciel et à des observations directes (développées avec des collègues
psychologues) nous pourrons mesurer, évaluer et analyser les processus mis en œuvre
dans l'apprentissage d'une langue.

Si ALGORD est un outil pour l'apprenant, il l'est également pour nous enseignants. Lors
des expériences menées auprès des élèves-ingénieurs de l'I.N.S.A., grâce aux capteurs
intégrés au logiciel et à des observations directes (développées avec des collègues
psychologues) nous pourrons mesurer, évaluer et analyser les processus mis en œuvre
dans l'apprentissage d'une langue.

Si ALGORD est un outil pour l'apprenant, il l'est également pour nous enseignants. Lors
des expériences menées auprès des élèves-ingénieurs de l'I.N.S.A., grâce aux capteurs
intégrés au logiciel et à des observations directes (développées avec des collègues
psychologues) nous pourrons mesurer, évaluer et analyser les processus mis en œuvre
dans l'apprentissage d'une langue.

Si ALGORD est un outil pour l'apprenant, il l'est également pour nous enseignants. Lors
des expériences menées auprès des élèves-ingénieurs de l'I.N.S.A., grâce aux capteurs
intégrés au logiciel et à des observations directes (développées avec des collègues
psychologues) nous pourrons mesurer, évaluer et analyser les processus mis en œuvre
dans l'apprentissage d'une langue.

Une des stratégies souvent recommandées est le classement par groupe ou catégorie, appelé par McDonough «associative clusters». Plusieurs expériences menées depuis les années 1950 ont prouvé l'efficacité de cette stratégie qu'Algord met en jeu en demandant à l'apprenant de classer ses données par thèmes et sous-thèmes.

Un autre élément dans la mémorisation est mis en valeur par P. Carré qui souligne qu'un individu fera davantage d'effort pour se souvenir de ce qui a de l'importance pour lui. La mémorisation exige la répétition, mais,

ce qui donne sa valeur aux aspects les plus répétitifs, conditionnant de l'apprentissage, c'est le degré de signification, la volonté de mémoriser, la motivation à apprendre et donc la liberté de choisir. (Carré 1991 : 180)

Cette liberté de choisir est respectée par ALGORD : la liberté d'enregistrer les mots/ expressions/règles qu'il souhaite apprendre, la liberté de n'utiliser que les éléments qui lui semblent utiles.

Utiliser ALGORD en mode acquisition peut aider l'apprenant à sélectionner et à organiser ses connaissances et ainsi faciliter leur mémorisation.

Utiliser ALGORD en mode exerciseur continue à jouer sur cette mémorisation.

Ellis et Sinclair, Thompson insistent sur l'importance de la révision régulière des acquisitions. Des recherches ont montré que le transfert de la mémoire à court terme dans la mémoire à long terme est facilité par des révisions espacées dans le temps. D'après Thompson,

self-testing (...) has been shown to improve long term recall ; [...] spaced practice leads to better long term recall. (in Wenden \& Rubin $1987: 47$ )

Les deux sont possibles grâce à l'exerciseur. De plus, le choix des exercices permet à l'apprenant de tester tous les éléments qu'il a rentrés dans le classeur.

En fait, l'apprenant a plusieurs façons de réviser : il peut tout simplement consulter les fiches, ou bien faire des exercices. Dans les deux cas, il a le choix entre réviser par thèmes pour bénéficier des " associations ", revoir les entrées depuis une certaine date, ou ne pas spécifier de critères, les données étant alors sélectionnées pour les exercices d'une façon aléatoire.

\section{Conclusion}

Nous avons essayé de montrer comment le logiciel ALGORD peut faciliter l'apprentissage d'une langue en proposant à l'apprenant différentes démarches qui, l'impliquant dans son apprentissage, l'amènent à une plus grande responsabilisation et, finalement, à une plus grande autonomie. 


\section{BIBLIOGRAPHIE}

Carré, P. 1991. Organiser l'apprentissage des langues étrangères. Paris : Les Éditions d'Organisation.

Dickinson, L. 1987. Self-instruction in Language Learning. Cambridge : Cambridge University Press.

Duda, R et P. Riley (dir.). 1990. Learning Styles. Collection Processus Discursifs. Nancy : Presses de Nancy.

Ellis, G. et B. Sinclair. 1989. Learning to Learn. Cambridge : Cambridge University Press.

Gairns, R. et S. Redman. 1990. Working with Words. Cambridge : Cambridge University Press.

Holec, H. 1981. Autonomy and Foreign language Learning. Oxford. Pergamon.

McDonough, S. 1981. Psychology in Foreign Language Learning. Londres : Allen and Unwin.

Stern, H. H. 1983. Fundamental Concepts of Language Teaching. Oxford : Oxford University Press

Wenden A. et J. Rubin. 1987. Learner Strategies and Language Learning. Englewood Cliffs : Prentice Hall International.

\section{NOTES}

1. Nous pourrions affiner considérablement cette grille pour en faire un moyen d'exploration pédagogique et imaginer à certaines intersections la possibilité de créer d'autres outils.

2. Plus tard, ALGORD sera disponible pour d'autres langues.

\section{AUTEURS}

\section{JEAN-PIERRE SOULA}

INSA Toulouse. soulajipe@voila.fr

\section{ANNE HERNANDEZ}

INSA Toulouse

\section{DANIEL MARRE}

INSA Toulouse 